\title{
A New Approach of Asphalt Quality Control: Inducting Online Supervision at Production Plant
}

\author{
Msc. Egli Dafa ${ }^{1 *}$ and Dr. Nissim Dafa ${ }^{2}$ \\ 1. Polytechinc University of Tirana, Albania \\ 2. Self-Employed
}

\begin{abstract}
According to the European standard EN 13108-21, quality control in an asphalt plant is based on the category of the plant. Category is established based on the results of the last 32 sample analysis. Depending on the category A and B, the minimum frequency of analysis in the finished product (sample per quantity) vary from 300-2,000 tons. Each analysis requires 2.5-3 kg asphalt. We believe that analyzing 2.5 or $3 \mathrm{~kg}$ of asphalt cannot represent 300 or 2,000 tons of production. The question is "What quality is the asphalt batch between two consecutive tests". Experience shows that there are fluctuations that deviate from the production parameters. For the reasons specified above, this research proposes a new method of quality control. This method suggests online automatic control and correction of the product, enabling transparency that leads to increased asphalt quality.
\end{abstract}

Key words: Asphalt, quality control, frequency, standard.

\section{Introduction}

All the Automated multi-process plant (AMPs) operate automatically and online, from the feed stage up to the production of asphalt. Throughout the process, a continuous printout is received of the production batches, fraction weights, bitumen temperature, bitumen weight and overall weight of the asphalt. This data are used to document the temperatures and weighting at different stages. A production batch is in effect a uniform mixture of mineral and filler materials and bitumen in the mixer, as a result of the feed of various weights of ingredients. The batch size is 1.5-4 tons depending on type of AMP.

Every plant has an internal control array that conducts daily tests of the quality of the raw materials, fillers, bitumen and the rest according to standards. This control restarts after several minutes of production when the laboratory receives a random sample from under the mixer. The analyzing process of a sample requires 2 hours. Producing 3 tons of

*Corresponding author: Egli Dafa, Msc., research field: civil engineering. asphalt takes approximately 50 seconds. This means that by the time that is required to perform the analysis, 432 tons of asphalt are produced, meaning that we can't possibly know the real quality of this product.

Because this control procedure is based only on "one sample in space", it doesn't consider the fluctuation of production parameters and possible deviations from the requirements that can happen in to the product segment between samples.

In cases where the results of analysis do not comply with the requirements of standard, laboratory should make appropriate corrections. If this analysis belongs to one or two batches with deviations in parameters according to requirements, and then returns to the previous settings, it is understood that this correction will lower the quality of asphalt for the entire period (time) until the results of second analysis.

To avoid making mistakes in the interpretation of the analyzed samples, laboratory technician before taking the sample should control the parameters registered in the printer of the factory, if there is large fluctuations for several minutes before and during the 
time he takes the sample for analysis. If these parameters correspond to points 1 and 2 (Graph 1) the sample represents the hole batch and the analyze should start. If the time of taking the sample coincide with the time that shows the arrow "a" (Graph 1) then the sample does not need to be analyzed because it is not representative. In this case the laboratory must also observe a few minutes of data printouts. If deviations continue he should take the following steps: 1) Check the material that comes from quarries. 2) Check the level of solid fractions in worm silos. If there are any faults found he should contact the production supervisor and halt the production. Afterwards there should be an investigation of the nets that filter the material, if any is broken, or either is blocked. If not he should check the scales which weight fractions that come down from worm silos.

Through the proposed program below, technicians can identify, immediately and continuously during production, a deviation from the standard, and even can also make suggestions on how to correct respective weight for particular factions, or the percentage of bitumen and temperature to meet standards.

According to the European Standard [2] the plants rating is determined based on the last 32 asphalt tests and their deviation from the required standard. In plants rated A, tests will be required every 600 and 2,000 tons, respectively. This sample of $3 \mathrm{~kg}$ should reflect a very large quantity of asphalt, which could be damaged. From studying production data of some plants were observed deviations of weights and temperatures required for the implementation of quality standards of asphalt. Graph 1 shows the parameters of asphalt production in a new. Out of the last 32 tests that were conducted, only one test was found to deviate from the standard, so the rating of the plant according to the European Standard [2] is A. According to the data received from the plant computer, it can be seen that there are only small deviations and according to the results of 2 tests which were taken during production (sample 1 and 2), it can be claimed that the asphalt complied with the demands of the European standard.

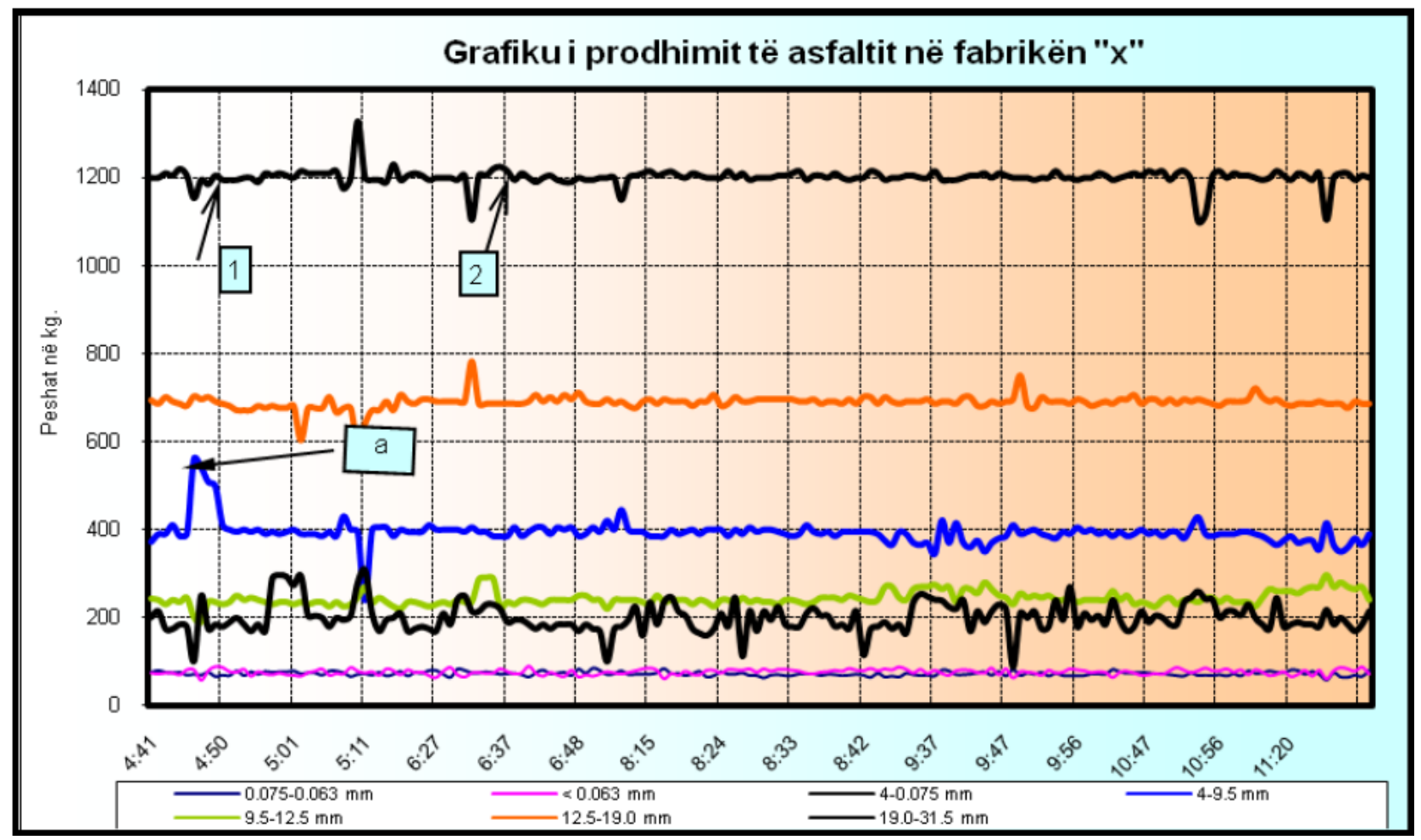

Graph. 1 Segmentation of weighting in new grade A plant. 
In testing conducted for evaluation of asphalt quality using the method proposed below, it turns out that in 513 tons asphalt, produced in 171 batches, there are 41 batches with deviations. Concretely 7 batches in which there is a deviation in fractions, 6 batches in which there is a deviation in bitumen percentage and 11 batches in which there is a deviation in temperature and therefore according to European Standards the manufacturer should be penalized in money.

\section{Methodology}

To achieve online survey of asphalt quality the process is described below:

\subsection{Calculation of Weight Deviation for Each Fraction and Bitumen}

Automatic calculation of the granulometry curves of each batch in view of real weights of each fraction as well as the total weight of batch. It automatically calculates the difference of values each fraction with the values defined by the JMF for each batch. When these differences deviate from the permitted values of set rates, for any amount of granulometry curve, the corresponding quadrant in Table 1 will change color and in the command center of the factory will be played a sound that informs the supervisor of the factory, when he is not watching the monitor, to getting the display color observed. This table shows the real values of bitumen percentage for each batch. These values and the overall weight of each batch were calculated real rates of bitumen for each batch. The corresponding vertical column of the Table 1 gives appropriate penalty calculated according to the norms by considering the differences size between the real values of bitumen percentage to that required under the JMF for each batch.

The same calculation is done for real temperatures and deviations by the temperature extreme values according to norms and also is calculate the penalty.

In a more complete way, quality control of the above parameters can be done by a program adapted for the command hall that will be shown in Fig. 1 in the computer screen of factory management. By this presentation the factory manager can make online corrections. In laboratory office must be displayed the same figure in order to achieve a better reconciliation of factory managers and laboratory technicians to increase the quality of asphalt.

\subsection{Weight Deviation Control and how to Correct when they Pass the Limit}

Every asphalt plant operates in a way that, despite fraction weight derivation from requirements of each batch, compensates by changing the weight of the next fraction so that all the batches are much more similar to each other. For any batch in production, by solving a series of equations is calculated the theoretical weight of each fraction in real time. These take into account the weight of every batch and the fraction granulometry that comes from the quarry, almost invariably.

It is done the difference between the estimated weight of each fraction with the actual weight for the same batch. With the results of the calculations is completed Table 2. With these data are built relevant graphs for each fraction and batch, in real time. Both of these graphics are shown below, the Graphs 2 and 3 . For each graph are set two horizontal yellow line on such values that weight below or above them, representing the weight of the granules in percentage, that passing the relevant sieve, which do not belong to the standard, thus requiring penalties.

As shown in Graph 2, difference of the real and calculated weights values for $4-5 \mathrm{~mm}$ fraction are symmetrical to the zero value, which shows that the required weight of this faction is completely safe to achieve the required standard. While in the Graph 3, it represents that fraction 9, 5-12 $\mathrm{mm}$ has a pronounced asymmetry to the zero line. In this case, should be added this faction by the factory technologist to achieve the required symmetry. 
Table 1 Online monitoring of asphalt temperature, aggregate fractions calculation, calculation of $\%$ of bitumen, and appropriate penalties for each batch production. Colored cells represent the deviation from the standard.

\begin{tabular}{|c|c|c|c|c|c|c|c|c|c|c|c|c|c|c|c|}
\hline \multirow{2}{*}{$\begin{array}{l}\text { Asphalt } \\
\text { Tep. }\end{array}$} & $\begin{array}{l}\text { Bitume } \\
\text { quantity \% }\end{array}$ & $4-7$ & $7-12$ & $12-20$ & $18-26$ & $28-38$ & $44-54$ & $60-70$ & $68-78$ & $84-94$ & 100 & $\begin{array}{l}\text { M. A. C. } \\
\text { Standards }\end{array}$ & \multirow{2}{*}{$\begin{array}{l}\text { Penalty for } \\
\text { granulometry } \\
\text { (ton) }\end{array}$} & \multirow{2}{*}{$\begin{array}{l}\text { Penalty for } \\
\text { bitumen \% } \\
\text { (ton) }\end{array}$} & \multirow{2}{*}{$\begin{array}{l}\text { Penalty } \\
\text { for Tep. } \\
\text { (ton) }\end{array}$} \\
\hline & 4.8 & 4.6 & 9 & 13 & 18 & 30 & 50 & 63 & 74 & 91 & 98 & $\overleftarrow{\text { G. M. F }}$ & & & \\
\hline 147 & 4.7 & 4.5 & 7.2 & 10.2 & 15.1 & 26.7 & 48.5 & 61.2 & 70.0 & 94.1 & 99.9 & $04: 42$ & 0.0 & 0.0 & 1.2 \\
\hline 150 & 4.7 & 4.5 & 7.3 & 10.3 & 15.2 & 26.7 & 48.5 & 61.1 & 70.0 & 94.1 & 99.9 & $04: 46$ & 0.0 & 0.0 & 0.9 \\
\hline 151 & 4.7 & 4.3 & 7.1 & 10.1 & 14.9 & 26.4 & 48.1 & 61.3 & 70.3 & 96.1 & 100 & $04: 47$ & 0.1 & 0.0 & 0.6 \\
\hline 153 & 4.6 & 4.4 & 7.1 & 10.0 & 14.8 & 26.1 & 47.5 & 60.5 & 68.9 & 93.2 & 99.9 & $06: 22$ & 0.1 & 0.0 & 0.3 \\
\hline 153 & 4.6 & 4.5 & 7.1 & 10.1 & 14.8 & 26.0 & 47.1 & 59.9 & 68.9 & 92.6 & 99.8 & 06:29 & 0.2 & 0.0 & 0.3 \\
\hline 155 & 4.6 & 4.3 & 6.9 & 9.8 & 14.5 & 25.8 & 47.1 & 59.7 & 68.5 & 92.2 & 99.8 & $06: 30$ & 0.9 & 0.0 & 0.0 \\
\hline 154 & 4.7 & 4.5 & 7.4 & 10.4 & 15.3 & 26.9 & 48.8 & 61.8 & 70.8 & 94.5 & 99.9 & 08:12 & 0.0 & 0.0 & 0.3 \\
\hline 154 & 4.6 & 4.4 & 7.3 & 10.3 & 15.0 & 26.4 & 47.8 & 60.2 & 68.8 & 92.6 & 99.8 & $08: 13$ & 0.1 & 0.0 & 0.3 \\
\hline 157 & 4.6 & 4.4 & 6.9 & 9.9 & 14.7 & 26.0 & 47.4 & 59.9 & 68.6 & 92.6 & 99.8 & $08: 16$ & 0.3 & 0.0 & 0.0 \\
\hline 159 & 4.7 & 4.3 & 6.9 & 9.9 & 14.7 & 26.1 & 47.6 & 60.2 & 69.2 & 93.1 & 99.9 & 08:18 & 0.1 & 0.0 & 0.0 \\
\hline 164 & 4.5 & 4.2 & 6.9 & 9.8 & 14.6 & 25.9 & 47.1 & 59.1 & 68.8 & 92.6 & 99.8 & $09: 34$ & 1.7 & 0.9 & 0.0 \\
\hline 161 & 4.5 & 4.4 & 7.0 & 9.9 & 14.7 & 25.9 & 47.1 & 59.1 & 69.0 & 92.8 & 99.8 & 09:38 & 0.0 & 0.9 & 0.0 \\
\hline \multirow[t]{2}{*}{161} & 4.5 & 4.3 & 7.1 & 10.0 & 14.7 & 25.9 & 46.9 & 58.9 & 68.5 & 92.5 & 99.8 & 09:40 & 0.1 & 0.9 & 0.0 \\
\hline & 4.5 & 4.4 & 7.3 & 10.2 & 15.0 & 26.3 & 47.6 & 59.6 & 69.3 & 93.1 & 99.9 & 09:44 & 0.0 & 0.9 & 0.0 \\
\hline 161 & 4.5 & 4.3 & 7.1 & 10.1 & 14.9 & 26.2 & 47.6 & 60.1 & 69.2 & 93.0 & 99.8 & 09:46 & 0.0 & 0.9 & 0.0 \\
\hline Total $=$ & & & & & & & & & & & & & 6.6 & 7.3 & 7.0 \\
\hline
\end{tabular}

Total production of the day $=586$ tons. Penalty total $=20.8$ tons or $3.5 \%$. 
Table 2 The automatic calculations of the differences between the actual weight and the theoretical weight in $\mathrm{kg}$ for each fraction and batch in real time.

\begin{tabular}{|c|c|c|c|c|c|c|}
\hline 19-31.5 & $12.5-19$ & $9.5-12.5$ & 4-9.5 & $0.075-4$ & $0.063-0.075$ & $<0.063$ \\
\hline-12 & 12 & -9 & -3 & 4 & 5 & -4 \\
\hline 26 & -10 & -1 & -7 & -12 & 2 & 0 \\
\hline 27 & 4 & -10 & -25 & -1 & 3 & 1 \\
\hline 16 & 6 & -6 & -2 & -22 & 6 & 0 \\
\hline 21 & 9 & -16 & -7 & -9 & -5 & 6 \\
\hline 100 & -8 & 30 & -174 & 51 & -4 & 4 \\
\hline-39 & 30 & 52 & -138 & 63 & 24 & 10 \\
\hline 33 & 15 & 3 & -111 & 56 & 1 & 3 \\
\hline 24 & 28 & 3 & -100 & 41 & -7 & 12 \\
\hline 21 & 6 & -1 & -27 & 3 & -10 & 6 \\
\hline 10 & 8 & -6 & -18 & -1 & -1 & 7 \\
\hline 2 & 23 & -20 & -10 & 7 & 0 & -3 \\
\hline 15 & 18 & -11 & -18 & -6 & -6 & 7 \\
\hline 29 & 13 & -18 & -16 & -15 & 8 & -2 \\
\hline 15 & 8 & -12 & -18 & 3 & -1 & 4 \\
\hline 29 & 9 & -8 & -10 & -23 & 3 & -3 \\
\hline
\end{tabular}

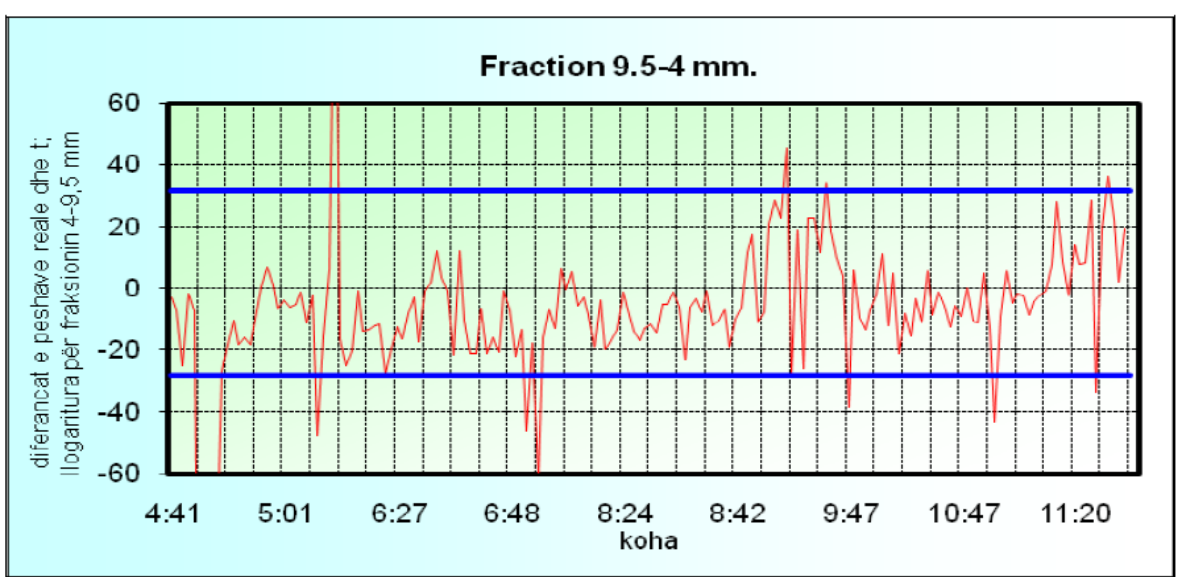

Graph. 2 Changes in weight of fraction 9.5-5.4 $\mathrm{mm}$ in time between calculated weighting and real weighting.

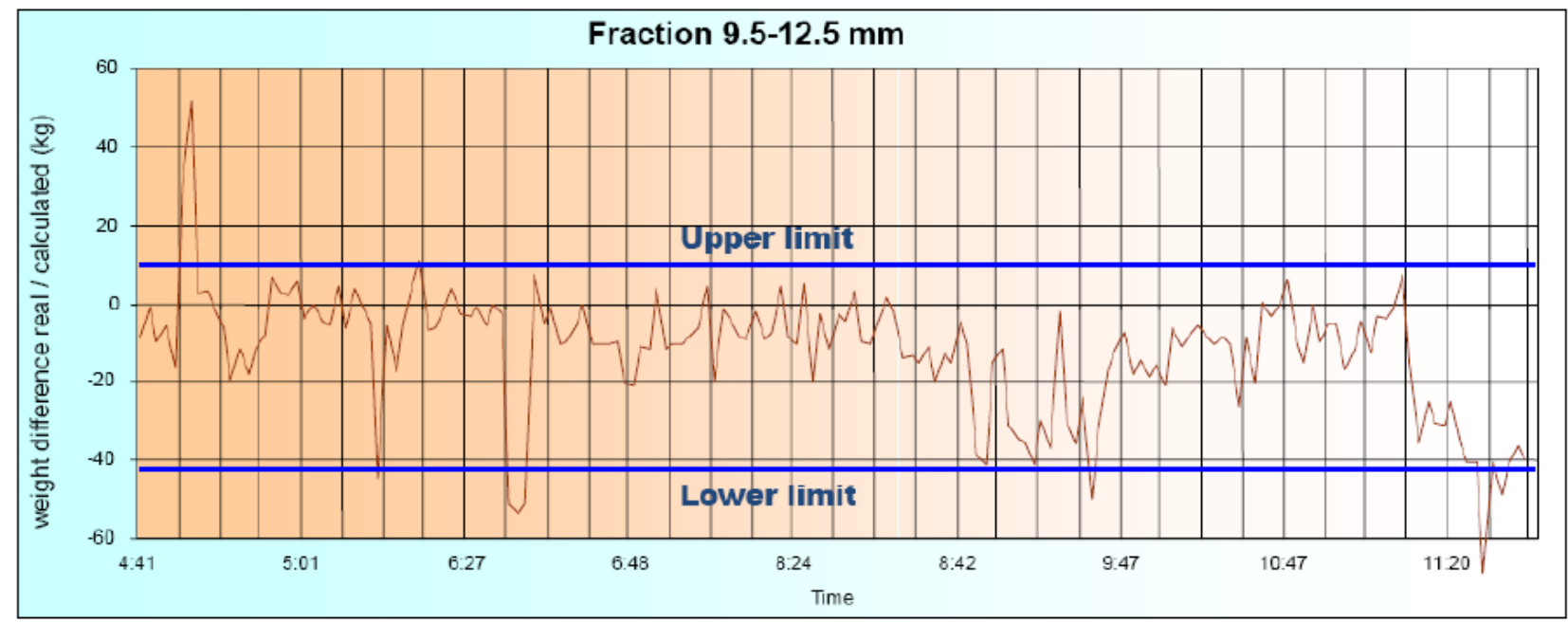

Graph. 3 Changes in weight of fraction $9.5-12.5 \mathrm{~mm}$ in time between calculated weighting and real weighting. 
All graphs for all fractions should shown constantly in the laboratory computer.

\subsection{The Factors that Can Cause Fraction Weight Deviation}

It is known that each quarries has its own specifications that are determined by geology (layers, chemical composition, etc.), types of crushers, extractors, dimensions, etc. When the raw material is homogeneous, we have a perfect balance between the amount of aggregate that enters the plant and quantity of asphalt produced. But it often happens that there are lithology changes of the quarry strata, in this case factory manager is required to add or reduce certain groups of aggregates by changing the speed of the conveyor belts until a new equilibrium is established. The same must be done even if the raw material comes from another quarry.

In factories producing asphalt that we have observed, the speeds of the conveyor belts are not registered. We believe that these speeds must be registered in the factory computer, because they are directly related to the changes of the fractions dimension entering the factory. With these data can be constructed a graphs with their speed. By setting on these graphics the speed upper and lower limits, that correspond the limit of fractions weights, will help to achieved the required standards. Their constant surveillance will help increasing the quality of asphalt.

Particular attention should be given to the speed changing of the conveyor belts, that depend on:

Incomplete separation of countries where aggregate fractions are stored. Blocking of the tank outputs by pieces of stones. The change of moisture, especially for fine fractions, which is more vulnerable to winter. Problems in various crushers etc.

\subsection{The Advantages of the Proposed Method}

The method proposed is very important, because it can drastically decrease the number of tests carried out for the final production. In this case we believe that is enough to be done only one test per day for any plant, since through it will be judged the accuracy of weighing of different factions and bitumen. This will allow the laboratory to have more time for all the other controls such as the quality of asphalt aggregates that require in the whole process of control.

It is understood that the reduction of the number of asphalt quality analysis will greatly impact on environmental protection. As known, the determination of the bitumen percentage in asphalt is made in two ways, through the extraction of bitumen with trichloroethylene and through the burning of the sample in special electric oven. In Albania is used the first method. For each extraction is used average three liter trichloroethylene. In the worst case, bitumen with trichloroethylene dissolved in it, is thrown outdoors, without thinking that the majority of trichloroethylene evaporates and leaves the atmosphere. It is well known that this product is disintegrant of ozone in the atmosphere.

In the best case this mixture is distilled in the laboratory, to extract trichloroethylene from bitumen. Even in this case around $30 \%$ during the distillation trichloroethylene passes in atmosphere, adding here and $10 \%$ of the solvent remaining in the aggregate who leaves the atmosphere during the process of worming the sample to be dried. Finally approximately $40 \%$ or 1.2 liter of trichloroethylene passes in atmosphere for every extraction.

I think in the computer command hall asphalt plant must be constantly shown, every 5 seconds current control schemes, which technologist are already familiar, and Table 1, Fig. 1, graphs with the differences between the estimated weight and the actual weight for each fraction.

Besides the usual way of plant control, after the implementation of the proposed method, (which is firmly based on the weights of mineral fractions), it will require more frequent calibration. 


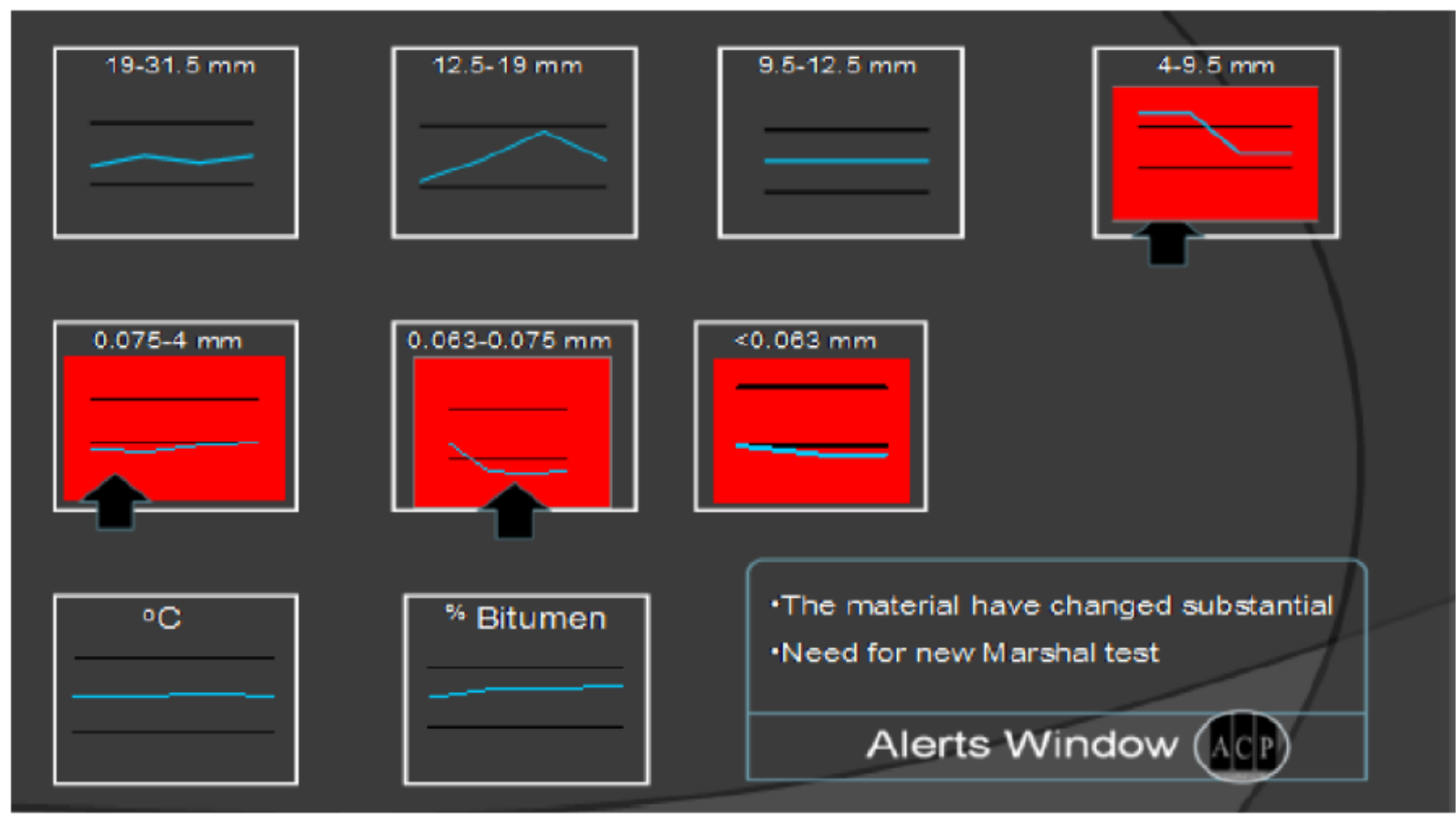

Fig. 1 Parameters shown before asphalt preparation. (Computer display, in control center).

\section{Conclusions}

Quality control of asphalt with the proposed method creates opportunities to correct in time the following parameters, leading to e better quality of product. This method provides the means of evaluating the work done by each plant worker. Controlling and correcting in real time the quality of produced asphalt can lessen the number of tests performed. This control is a great way to assess the reliability level of the factory and creates high premises that a sample of $3 \mathrm{~kg}$ can represent an asphalt production of 2,000 tons. The data collected from 12 plants was elaborated with the proposed program. The results showed that there were penalties from $2 \%$ to $7 \%$ for any amount of asphalt produced, to be applied, while the actual analysis showed no penalties. After applying this method for several days in an asphalt plant, there was a reduction of batches that were not in accordance to the required normative, by $4.8 \%$.

\section{References}

[1] Revêtement de Chaussée en Enrobe (Pavement Coating). 2003. (Control Test, Section 13, page 9-19). Transports Québec CCDG.

[2] Asphalt Quality Control Procedure in Several Israel Asphalt Plants.

[3] Bituminous Mixtures. 2006. Material Specification. Factory Production Control. EN 13108-21.

[4] Production Datasheets of 12 Asphalt Plants. (Israel and Albanian plants). 